\title{
PROBABILISTIC SQUARE FUNCTIONS AND A PRIORI ESTIMATES
}

\author{
BY \\ ANDREW G. BENNETT
}

\begin{abstract}
We obtain a priori estimates for Riesz transforms and their variants, that is, estimates with bounds independent of the dimension of the space and/or the nature of the boundary. The key to our results is to give probabilistic definitions which do not depend on the geometry of the situation for the transformations in question. We then use probabilistic square functions to prove our a priori estimates.
\end{abstract}

The purpose of this paper is to show how probabilistic methods can be used to obtain a priori estimates for Riesz transforms and their variants, that is, estimates with bounds independent of the dimension of the space and/or the nature of the boundary. We shall prove our results for domains with smooth boundaries. The importance of estimates, with bounds independent of domain, for domains with smooth boundaries is that one can prove corresponding results for more general domains using limiting arguments. We shall not deal with these limiting arguments in this paper. In Theorem 1, we prove an estimate for the boundary values of a system of conjugate harmonic functions; in Theorem 2 , we show how a variation on this proof allows us to give an alternate proof of the $L^{p}$ boundedness, independent of dimension, of the Riesz transform in $R^{n}$; and in Theorem 3 , we prove an estimate for an appropriate analogue of the $g^{*}$ function for general domains.

The approach used here was suggested to the author by E. M. Stein. This paper represents part of the author's work on his dissertation.

Our basic tools will be probabilistic square functions; the following is the basic example. Let $D$ be a domain in $R^{n}$. Let $B_{t}$ be Brownian motion in $D$ and let $\tau$ be a stopping time. If $u$ is harmonic in $D$, then $u\left(B_{t}\right)$ is a martingale and we can define the martingale maximal function, $u^{*}$, and martingale square function, $S(u)$, to be the following random variables.

DEFINITION 1. $u^{*}=\sup _{0 \leq t \leq \tau} u\left(B_{t}\right), S(u)=\left(u^{2}\left(B_{0}\right)+\int_{0}^{\tau}\left|\nabla u\left(B_{t}\right)\right|^{2} d t\right)^{1 / 2}$.

It should be noted that the integral in the definition of $S(u)$ is the quadratic variation of the martingale $u\left(B_{t}\right)$, i.e. $u^{2}\left(B_{t}\right)-u^{2}\left(B_{0}\right)-\int_{0}^{t}\left|\nabla u\left(B_{s}\right)\right|^{2} d s$ is a martingale. It follows that $E\left[u^{2}\left(B_{\tau}\right)\right]=E\left[S^{2}(u)\right]$. We now have the following lemmas.

Lemma 1 (DoOB). For $1<p<\infty$

$$
((p-1) / p)^{p} E\left[u^{* p}\right] \leq E\left[\left|u\left(B_{\tau}\right)\right|^{p}\right] \leq E\left[u^{* p}\right] .
$$

ProOF. See [2].

Received by the editors November 30, 1984 .

1980 Mathematics Subject Classification. Primary 42A36; Secondary $60 \mathrm{~J} 65$. 
LEMMA 2 (BURKHOLDER). For $1<p<\infty$, there exist $c_{p}$ and $C_{p}$, independent of $D$ and $n$, such that

$$
c_{p} E\left[S(u)^{p}\right] \leq E\left[u^{* p}\right] \leq C_{p} E\left[S(u)^{p}\right] .
$$

ProOF. See $[\mathbf{1}]$.

LEMMA 3 (KAKUTANI). Let $D$ be a bounded domain in $R^{n}$ with smooth boundary and let $x^{0} \in D$. If $B_{t}$ is Brownian motion in $D$ with $B_{0} \equiv x^{0}$, then the probability distribution of the point where $B_{t}$ first hits $\partial D$ is harmonic measure on $\partial D$ associated to $x^{0}$.

ProOf. See $[\mathbf{5}, \mathbf{6}]$.

COROllary 1. Suppose D, $x^{0}$ and $B_{t}$ are as in Lemma 3. If we let $\tau$ be the first hitting time of $B_{t}$ on $\partial D$ and $\|f\|_{p}^{p}=\int_{\partial D}|f(x)|^{p} d \mu(x)$, where $d \mu$ is harmonic measure on $\partial D$ associated to $x^{0}$, then for $1<p<\infty$, there exist $b_{p}$ and $B_{p}$, independent of $D$ and $n$, such that

$$
b_{p} E\left[S^{p}\left(u_{j}\right)\right]^{1 / p} \leq\left\|f_{j}\right\|_{p} \leq B_{p} E\left[S^{p}\left(u_{j}\right)\right]^{1 / p} .
$$

PROOF. Trivial consequence of Lemmas 1,2 and 3.

These lemmas and particularly Corollary 1 are the keys to relating our probabilistic square functions to our a priori estimates.

Our first theorem involves the boundary values of a system of conjugate harmonic functions, what we shall call a system of Riesz transforms.

DEFINITION 2. Let $D$ be a bounded domain in $R^{n}$ with smooth boundary and let $x^{0} \in D$. Suppose $f_{1}, \ldots, f_{n}$ are $L^{2}$ functions on $\partial D$ and let $u_{1}, \ldots, u_{n}$ be the solutions of the Dirichlet problems, $\Delta u_{j}=0$ and $\left.u_{j}\right|_{\partial D}=f_{j}$. We say $f_{1}, \ldots, f_{n}$ is a system of Riesz transforms in case $u_{1}, \ldots, u_{n}$ forms a system of conjugate harmonic functions, i.e. they satisfy the generalized Cauchy-Riemann equations (see [7])

$$
\frac{\partial u_{j}}{\partial x_{k}}=\frac{\partial u_{k}}{\partial x_{j}}, \quad \sum_{j=1}^{n} \frac{\partial u_{j}}{\partial x_{j}}=0, \quad u_{1}\left(x^{0}\right)=\cdots=u_{n}\left(x^{0}\right)=0 .
$$

The last condition is necessary for general $D$ since otherwise the Riesz transforms would only be determined up to an additive constant. (Note: We could use the condition $u_{1}\left(x^{0}\right)=\cdots=u_{n}\left(x^{0}\right)$, not necessarily $=0$, and all our conclusions would still hold. The case where $u_{j}\left(x^{0}\right)=0$ is just simpler.) Next, let $\|f\|_{p}^{p}=$ $\int_{\partial D}|f(x)|^{p} d \mu(x)$, where $d \mu$ is harmonic measure on $\partial D$ associated to $x^{0}$, and let $\sum_{i}^{\prime}$ stand for $\sum_{i=1}^{n}$, where $i \neq j$.

THEOREM 1. If $f_{1}, \ldots, f_{n}$ is a system of Riesz transforms with respect to $x^{0}$, then for $1<p<\infty$, there exists $A_{p}$ independent of $D$ and $n$ such that $\left\|f_{j}\right\|_{p} \leq$ $A_{p} \sum_{i}^{\prime}\left\|f_{i}\right\|_{p}$.

PROOF. Here we shall use everything as defined in Corollary 1. By Corollary 1 , if we can show $S\left(u_{j}\right) \leq \sum_{i}^{\prime} S\left(u_{i}\right)$, we will be done. To simplify the notation, let

$$
A_{k}^{i}=\left(\int_{0}^{\tau}\left|\frac{\partial u_{i}}{\partial x_{k}}\left(B_{t}\right)\right|^{2} d t\right)^{1 / 2}
$$


Then we have

$$
\begin{aligned}
& S^{2}\left(u_{j}\right)=\int_{0}^{\tau}\left|\nabla u_{j}\left(B_{t}\right)\right|^{2} d t \\
& =\sum_{i}^{\prime}\left(\int_{0}^{\tau}\left|\frac{\partial u_{j}}{\partial x_{i}}\left(B_{t}\right)\right|^{2} d t\right)+\int_{0}^{\tau}\left|\frac{\partial u_{j}}{\partial x_{j}}\left(B_{t}\right)\right|^{2} d t \\
& =\sum_{i}^{\prime}\left(\int_{0}^{\tau}\left|\frac{\partial u_{i}}{\partial x_{j}}\left(B_{t}\right)\right|^{2} d t\right)+\int_{0}^{\tau}\left|\sum_{i}^{\prime} \frac{\partial u_{i}}{\partial x_{i}}\left(B_{t}\right)\right|^{2} d t \\
& =\sum_{i}^{\prime}\left(A_{j}^{i}\right)^{2}+\sum_{i}^{\prime}\left(A_{i}^{i}\right)^{2}+2 \sum_{i}^{\prime} \sum_{k>i}^{\prime}\left[\int_{0}^{\tau}\left|\frac{\partial u_{i}}{\partial x_{i}}\left(B_{t}\right) \frac{\partial u_{k}}{\partial x_{k}}\left(B_{t}\right)\right| d t\right] \\
& \leq \sum_{i}^{\prime}\left(\left(A_{j}^{i}\right)^{2}+\left(A_{i}^{i}\right)^{2}\right)+2 \sum_{i}^{\prime} \sum_{k>i}^{\prime}\left[\left(\int_{0}^{\tau}\left|\frac{\partial u_{i}}{\partial x_{i}}\left(B_{t}\right)\right|^{2} d t\right)^{1 / 2}\right. \\
& \left.\times\left(\int_{0}^{\tau}\left|\frac{\partial u_{k}}{\partial x_{k}}\left(B_{t}\right)\right|^{2} d t\right)^{1 / 2}\right] \\
& =\sum_{i}^{\prime}\left(\left(A_{j}^{i}\right)^{2}+\left(A_{i}^{i}\right)^{2}\right)+2 \sum_{i}^{\prime} \sum_{k>i}^{\prime} A_{i}^{i} A_{k}^{k} \\
& \leq \sum_{i}^{\prime}\left(\left(A_{j}^{i}\right)^{2}+\left(A_{i}^{i}\right)^{2}\right)+2 \sum_{i}^{\prime} \sum_{k>i}^{\prime}\left[\left(\left(A_{i}^{i}\right)^{2}+\left(A_{j}^{i}\right)^{2}\right)^{1 / 2}\left(\left(A_{k}^{k}\right)^{2}+\left(A_{j}^{k}\right)^{2}\right)^{1 / 2}\right] \\
& =\left[\sum_{i}^{\prime}\left(\left(A_{j}^{i}\right)^{2}+\left(A_{i}^{i}\right)^{2}\right)^{1 / 2}\right]^{2} \\
& =\left[\sum_{i}^{\prime}\left(\int_{0}^{\tau}\left|\frac{\partial u_{i}}{\partial x_{j}}\left(B_{t}\right)\right|^{2} d t+\int_{0}^{\tau}\left|\frac{\partial u_{i}}{\partial x_{i}}\left(B_{t}\right)\right|^{2} d t\right)^{1 / 2}\right]^{2} \\
& \leq\left[\sum_{i}^{\prime}\left(\int_{0}^{\tau}\left|\nabla u_{j}\left(B_{t}\right)\right|^{2} d t\right)^{1 / 2}\right]^{2}=\left[\sum_{i}^{\prime} S\left(u_{i}\right)\right]^{2} \text {. }
\end{aligned}
$$

So $S\left(u_{j}\right) \leq \sum_{i}^{\prime} S\left(u_{i}\right)$ and the theorem is proved.

Next, we give an example to show that in the inequality $\left\|f_{j}\right\|_{p}^{p} \leq A_{p} \sum_{i}^{\prime}\left\|f_{i}\right\|_{p}^{p}$, the bound $A_{p}$ grows with $n$. We let $D$ be the unit ball in $R^{n}$ and $x^{0}=0$. Let

$$
\begin{aligned}
u_{1}(x) & =(n-1) x_{1}, \\
u_{2}(x) & =-x_{2}, \\
\vdots & \\
u_{n}(x) & =-x_{n} .
\end{aligned}
$$

Then $u_{1}, \ldots, u_{n}$ forms a system of conjugate harmonic functions and hence the $f_{j}=\left.u_{j}\right|_{\partial D}$ form a system of Riesz transforms. Let $\alpha_{p}=\int_{\partial D}\left|x_{1}\right|^{p} d \sigma(x)$, where $d \sigma(x)$ is Lebesgue measure on the surface of the unit ball in $R^{n}$ normalized to have 
total mass 1 (and also harmonic measure with respect to $x^{0}=0$ ). Then we have

$$
\begin{aligned}
\left\|f_{1}\right\|_{p}^{p} & =(n-1)^{p} \int_{\partial D}\left|x_{1}\right|^{p} d \sigma(x)=(n-1)^{p} \alpha_{p}, \\
\left\|f_{2}\right\|_{p}^{p} & =\int_{\partial D}\left|x_{2}\right|^{p} d \sigma(x)=\alpha_{p} \\
\vdots & \\
\left\|f_{n}\right\|_{p}^{p} & =\int_{\partial D}\left|x_{n}\right|^{p} d \sigma(x)=\alpha_{p} .
\end{aligned}
$$

So $\left\|f_{1}\right\|_{p}^{p}=(n-1)^{p} \alpha_{p}$ but $\sum_{i=2}^{n}\left\|f_{i}\right\|_{p}^{p}=(n-1) \alpha_{p}$ and so $A_{p}$ must grow with $n$.

In our second theorem, we show how a variation of these techniques can be applied to the domain $R_{+}^{n+1}$ and given an alternate proof of the $L^{p}$ boundedness of the Riesz transform with bound independent of dimension.

Let $f \in L^{2}\left(R^{n}\right)$. Then we define the Riesz transforms of $f$ to be the functions $f_{j}$ in $L^{2}$ such that $f_{j}^{\wedge}(\xi)=\left(i \xi_{j}\right) /|\xi| f^{\wedge}(\xi)$, where $f^{\wedge}(\xi)$ is the Fourier transform of $f(x)$. By convention, we shall call $x_{0}=y$ and $f_{0}(x)=f(x)$. Then if $\Delta u_{j}=0$ in $R_{+}^{n+1}$ and $\left.u_{j}\right|_{R^{n}}=f_{j}$, the $u_{j}$ are a system of conjugate harmonic equations and satisfy the generalized Cauchy-Riemann equations given in Definition 2 with $x^{0}=\infty$. We write $R(f)=\left(f_{1}, \ldots, f_{n}\right)$ and then $|R(f)|^{2}=f_{1}^{2}+\cdots+f_{n}^{2}$. Here we use $\|f\|_{p}$ for the usual $L^{p}$ norm on $R^{n}$. To simplify the notation, we write $\|R(f)\|_{p}$ for $\||R(f)|\|_{p}$.

THEOREM 2 (STEIN). For $1<p<\infty$, there exists $A_{p}$, independent of $n$, such that $\|R(f)\|_{p} \leq A_{p}\|f\|_{p}$.

PROOF. We will first introduce a variation on Brownian motion due to Gundy and Varopoulos called "background radiation" which we need to deal with $R_{+}^{n+1}$.

DEFINITION 3. Background radiation is the continuous process $Z_{t}=\left(X_{t}, Y_{t}\right)$, $-\infty<t<0$, taking values in $R_{+}^{n+1}$ and having the following properties:

(1) $Y_{-\infty}=\lim _{t \downarrow-\infty} Y_{t}=+\infty$.

(2) $Y_{0}=\lim _{t \uparrow 0} Y_{t}=0$ and $Z_{0}=\lim _{t \uparrow 0} Z_{t}$ exists as a point on the boundary $R^{n}$.

(3) If, for $a>0$, we let $T^{a}=\inf \left\{t: Y_{t}=a\right\}$, then $Z_{t}^{a}=Z_{T^{a_{+}}}, 0 \leq t \leq-T^{a}$ is a copy of standard Brownian motion on $R_{+}^{n+1}$ with initial distribution being Lebesgue measure on the level $\{y=a\}$ and which terminates upon hitting the boundary $R^{n}$.

See [4] for an explicit construction of this process. It should be noted that $Z_{t}$ is not itself Brownian motion.

With this process, we are now ready to define our variations on $S(u)$ for $R_{+}^{n+1}$. If $f \in L^{2}$ and $\Delta u=0$ in $R_{+}^{n+1}$ with $\left.u\right|_{R^{n}}=f$, we define

DEFINITION 4.

$$
\mathbf{S}(f)=\left(\int_{-\infty}^{0}\left|\nabla u\left(Z_{t}\right)\right|^{2} d t\right)^{1 / 2}, \quad \mathbf{S}_{1}(f)=\left(\int_{-\infty}^{0}\left|\frac{\partial u}{\partial y}\left(Z_{t}\right)\right|^{2} d t\right)^{1 / 2}
$$

We shall also need a special definition for the vector-valued case.

Definition $4^{\prime} . \mathbf{S}_{1}(R(f))=\left(\mathbf{S}_{1}^{2}\left(f_{1}\right)+\cdots+\mathbf{S}_{1}^{2}\left(f_{n}\right)\right)^{1 / 2}$.

Our theorem will now follow from the next three lemmas. 
Lemma 4. For $1<p<\infty, E\left[\mathbf{S}_{1}^{p}(R(f))\right] \leq E\left[\mathbf{S}^{p}(f)\right]$.

Lemma 5. For $1<p<\infty$, there exist $a_{p}, A_{p}$, independent of $n$, such that

$$
a_{p} E\left[\mathbf{S}^{p}(f)\right] \leq\|f\|_{p}^{p} \leq A_{p} E\left[\mathbf{S}^{p}(f)\right] .
$$

Lemma 6. For $1<p<\infty$, there exists $B_{p}$, independent of $n$, such that

$$
\|R(f)\|_{p}^{p} \leq B_{p} E\left[\mathbf{S}_{1}^{p}(R(f))\right] .
$$

Proof OF LEMMA 4. This follows trivially from the generalized CauchyRiemann equations.

Proof OF LEMMA 5. Let $\mathbf{S}_{(b)}=\left(u^{2}\left(Z_{0}^{b}\right)+\int_{0}^{-T^{b}}\left|\nabla u\left(Z_{t}^{b}\right)\right|^{2} d t\right)^{1 / 2}$ and observe that Lemmas 1 and 2 imply that for $1<p<\infty$, there exist $a_{p}, A_{p}$, independent of $n$, such that

$$
a_{p} E\left[\mathbf{S}_{(b)}^{p}(f)\right] \leq E\left[u^{p}\left(Z_{-T^{b}}^{b}\right)\right] \leq A_{p} E\left[\mathbf{S}_{(b)}^{p}(f)\right] .
$$

Also note that $E\left[u^{p}\left(Z_{-T^{b}}^{b}\right)\right]=E\left[u^{p}\left(Z_{0}\right)\right]=\|f\|_{p}^{p}$ by the definition of background radiation. Lemma 5 follows by combining these observations and letting $b \rightarrow+\infty$.

PROOF OF LEMMA 6. We first prove Lemma 6 in the scalar valued case. The first step is to show that $\|f\|_{2}^{2}=4 E\left[S_{1}^{2}(f)\right]$. As is well known, if we take the Fourier transform of $u(x, y)$ in the $x$ variables only, we obtain $u^{\wedge}(\xi, y)=e^{-2 \pi|\xi| y} f^{\wedge}(\xi)$. So by Plancherel's theorem, we can see that $2\|(\partial u / \partial y)(\cdot, y)\|_{2}=\||\nabla u(\cdot, y)|\|_{2}$ from which it follows that $4 E\left[\mathbf{S}_{1}^{2}(f)\right]=E\left[\mathbf{S}^{2}(f)\right]$. But $E\left[\mathbf{S}_{(b)}^{2}(f)\right]=E\left[u^{2}\left(Z_{0}\right)\right]=$ $\|f\|_{2}^{2}$, and by letting $b \rightarrow+\infty$, we see that $E\left[\mathbf{S}^{2}(f)\right]=\|f\|_{2}^{2}$. So we have our identity $\|f\|_{2}^{2}=4 E\left[\mathbf{S}_{1}^{2}(f)\right]$. Polarizing this identity, we obtain $\int_{R^{n}} f(x) g(x) d x=$ $4 E\left[\mathbf{S}_{1}(f) \mathbf{S}_{1}(g)\right]$ for $f, g \in L^{2}\left(R^{n}\right)$. Let $\Phi=\left\{g \in L^{2} \cap L^{q}:\|g\|_{q}=1\right\}$, where $q$ is the conjugate exponent to $p, 1<p<\infty$. Then

$$
\begin{aligned}
\|f\|_{p} & =\inf _{g \in \Phi} \int_{R^{n}} f(x) g(x) d x=4 \inf _{g \in \Phi} E\left[\mathbf{S}_{1}(f) \mathbf{S}_{1}(g)\right] \\
& \leq 4\left(\inf _{g \in \Phi} E\left[\mathbf{S}_{1}^{q}(g)\right]^{1 / q}\right) E\left[\mathbf{S}_{1}^{p}(f)\right]^{1 / p} \leq \frac{4}{a_{q}} E\left[\mathbf{S}_{1}^{p}(f)\right]^{1 / p},
\end{aligned}
$$

where we have used Hölder's inequality in the third step and the fact that

$$
E\left[\mathbf{S}_{1}^{q}(g)\right]^{1 / q} \leq E\left[\mathbf{S}^{q}(g)\right]^{1 / q} \leq\|g\|_{q} / a_{q}
$$

in the fourth step. This establishes Lemma 6 in the scalar-valued case. We now note that the exact same proof works for the vector-valued case and our proof of Lemma 6 is finished if we substitute $R(f)$ for $f$. Theorem 2 now follows trivially from our three lemmas.

Our probabilistic proof closely parallels Stein's analytic proof $($ see $[\mathbf{8}, \mathbf{9}])$. Our probabilistic square functions replace the Littlewood-Paley $g$-functions in Stein's proof and our three lemmas are exact analogues of the three lemmas in Stein's proof.

Our final theorem involves the square function $g^{*}(f)(z) \cdot g^{*}(f)(z)$ is defined on $R^{n}$ as follows.

DEFinition 5. Let $f \in L^{2}\left(R^{n}\right)$ and let $u$ be the Poisson integral of $f$. Then

$$
\left(g^{*}(f)(z)\right)^{2}=\int_{0}^{\infty} \int_{R^{n}} y^{2}\left(|z-x|^{2}+y^{2}\right)^{-(n+1) / 2}|\nabla u(x, y)|^{2} d x d y
$$


With this definition, it can be shown that $\left\|g^{*}(f)\right\|_{p} \leq A_{p}\|f\|_{p}$ for $2 \leq p<\infty$ with $A_{p}$ independent of $n$ (see [7]). We shall extend both the definition of $g^{*}(f)$ and this last inequality to general domains (the idea for extending the definition to general domains is due to Kenig).

Let $D$ be a bounded domain in $R^{n}$ with smooth boundary and let $x^{0} \in D$. Let $u$ be harmonic in $D$ and $\left.u\right|_{\partial D}=f$. Let $G(x, y)$ be the Green's function for $D$ and let $P(x, y)$ be the Poisson kernel for $D$.

DEFINITION 6.

$$
\left(g^{*}(f)(z)\right)^{2}=\int_{D} \frac{\left|G\left(x^{0}, y\right)\right|}{P\left(x^{0}, z\right)} P(y, z)|\nabla u(y)|^{2} d y \quad(z \in \partial D) .
$$

It should be noted that if we take $D=R_{1}^{n+1}$ and let $x^{0} \rightarrow+\infty$, we get the same definition for $g^{*}(f)$ in Definition 6 as was given in Definition 5 , up to a multiplicative constant. Now let $\|f\|_{p}^{p}=\int_{\partial D}|f(x)|^{p} d \mu(x)$, where $d \mu(x)$ is harmonic measure on $\partial D$ with respect to $x^{0}$. (Note that $d \mu(x)=P\left(x^{0}, x\right) d \sigma(x)$, where $d \sigma(x)$ is Lebesgue measure on $\partial D$.)

THEROEM 3. If $u\left(x^{0}\right)=0$, then for $2 \leq p<\infty$, there exists $A_{p}$, independent of $D$ and $n$, such that $\left\|g^{*}(f)\right\|_{p} \leq A_{p}\|f\|_{p}$.

PROOF. The key to our proof is to give a probabilistic definition of $g^{*}(f)$ in terms of conditional expectation. If, as before, $B_{t}$ is Brownian motion on $D$, with $B_{0} \equiv x^{0}$, and $B_{t}$ stopped at $\partial D$ at time $\tau$, then we will show $\left(g^{*}(f)(z)\right)^{2}=$ $\frac{1}{2} E\left[\int_{0}^{\tau}\left|\nabla u\left(B_{t}\right)\right|^{2} d t \mid B_{\tau}=z\right]$. We start by defining the occupation time for $B_{t}$. For measurable $A \subset D$, the occupation time is the random variable $\int_{0}^{\tau} \chi_{A}\left(B_{t}\right) d t$, where $\chi_{A}$ is the indicator function of $A$. We can also define a measure $\nu$ on $D$ by the expected value of the occupation time, i.e. $\nu(A)=E\left[\int_{0}^{\tau} \chi_{A}\left(B_{t}\right) d t\right]$. Since $B_{t}$ is stopped at $\partial D, B_{t} \in \partial D$ for $t \geq \tau, B_{t} \notin D$ for $t \geq \tau$, and we could just as well define $\nu(A)=E\left[\int_{0}^{\infty} \chi_{A}\left(B_{t}\right) d t\right]$. The importance of the measure $\nu$ is that $\int_{D} f(x) d \nu(x)=E\left[\int_{0}^{\tau} f\left(B_{t}\right) d t\right]$. We can show this by first using simple $f$ and then taking limits. Our next observation is that

$$
\nu(A)=E\left[\int_{0}^{\infty} \chi_{A}\left(B_{t}\right) d t\right]=\int_{0}^{\infty} E\left[\chi_{A}\left(B_{t}\right)\right] d t=\int_{0}^{\infty} P_{t}\left(x^{0}, A\right) d t,
$$

where $P_{t}\left(x^{0}, A\right) \equiv E\left[\chi_{A}\left(B_{t}\right)\right]$ is the probability of a transition from $x^{0}$ to $A$ in time $t$. For Brownian motion, $P_{t}\left(x^{0}, A\right)$ has a density $p_{t}\left(x^{0}, y\right)$ and $\nu(A)$ also has a density $v(y)=\int_{0}^{\infty} p_{t}\left(x^{0}, y\right) d t$. We have the following lemma.

LEMMA 7. $v(y)=2\left|G\left(x^{0}, y\right)\right|$.

ProOF. This follows from the fact that if $w(x)=E\left[\int_{0}^{\tau} f\left(B_{t}\right) d t \mid B_{0}=x\right]$, then $\frac{1}{2} \Delta w=-f$ and $w(x)=0$ on $\partial D$ (see $[\mathbf{3}]$ ).

Next, we look at the transition density for the process conditioned to hit $\partial D$ at $z, p_{t}^{(z)}\left(x^{0}, y\right)$. By Bayes' theorem,

$$
p_{t}^{(z)}\left(x^{0}, y\right)=\frac{P(y, z)}{P\left(x^{0}, z\right)} p_{t}\left(x^{0}, y\right)
$$


remembering that $P\left(x^{0}, z\right)$ is the density of $B_{\tau}$ given $B_{0} \equiv x^{0}$. Thus, the occupation density for the conditioned process is given by

$$
\begin{aligned}
v^{(z)}(y) & =\int_{0}^{\infty} p_{t}^{(z)}\left(x^{0}, y\right) d t=\int_{0}^{\infty} \frac{P(y, z)}{P\left(x^{0}, z\right)} p_{t}\left(x^{0}, y\right) d t \\
& =\frac{P(y, z)}{P\left(x^{0}, z\right)} v(y)=\frac{P(y, z)}{P\left(x^{0}, z\right)} 2\left|G\left(x^{0}, y\right)\right|
\end{aligned}
$$

So finally

$$
\begin{aligned}
\left(g^{*}(f)(z)\right)^{2} & =\int_{D} \frac{\left|G\left(x^{0}, y\right)\right|}{P\left(x^{0}, z\right)} P(y, z)|\nabla u(x, y)|^{2} d x d y \\
& =\frac{1}{2} E\left[\int_{0}^{\tau}\left|\nabla u\left(B_{t}\right)\right|^{2} d t \mid B_{\tau}=z\right] .
\end{aligned}
$$

Now, remembering from Lemma 3 that the distribution of $B_{\tau}$ is harmonic measure on $\partial D$, we have for $2 \leq p<\infty$

$$
\begin{aligned}
\left\|g^{*}(f)\right\|_{p}^{p} & =\frac{1}{2} E\left[E\left[\int_{0}^{\tau}\left|\nabla u\left(B_{t}\right)\right|^{2} \mid B_{\tau}=z\right]^{(p / 2)}\right] \\
& \leq \frac{1}{2} E\left[E\left[\left(\int_{0}^{\tau}\left|\nabla u\left(B_{t}\right)\right|^{2} d t\right)^{(p / 2)} \mid B_{\tau}=z\right]\right] \\
& =\frac{1}{2} E\left[\left(\int_{0}^{\tau}\left|\nabla u\left(B_{t}\right)\right|^{2} d t\right)^{(p / 2)}\right] \\
& =\frac{1}{2} E\left[S^{p}(u)\right] \leq A_{p}\|f\|_{p}^{p} .
\end{aligned}
$$

Here, we have used Jensen's inequality in step 2 and the fact that $u\left(x^{0}\right)=0$ in step 4.

We close with an example to show that $p \geq 2$ is the best exponent we can get in Theorem 3. Let $h(x)=|x|^{-(n+1) / 2}$ for $x \in R^{n}$. Let $v(x, y)$ be the Poisson integral for $R_{+}^{n+1}$ of $h$. Then since $h$ is homogeneous of degree $-(n+1) / 2, v$ is homogeneous of degree $-(n+1) / 2$ and $|\nabla v|$ is homogeneous of degree $-(n+3) / 2$. A calculation shows $v(0, y)=c_{n} y^{-(n+1) / 2}$ and hence

$$
\frac{\partial v}{\partial y}\left(0, y_{0}\right)=c_{n}^{\prime} y_{0}^{-(n+3) / 2} \text {. }
$$

So $|\nabla v(x, y)| \geq c_{n}^{\prime}\left(|x|^{2}+y^{2}\right)^{-(n+3) / 2}$ for $(x, y)=\left(0, y_{0}\right)$ and, by continuity, this inequality must hold in some neighborhood about $\left(0, y_{0}\right)$. Finally, by homogeneity, this inequality must hold in some cone of the form $\{(x, y):|x| \leq \alpha y\}$ with $\alpha>0$. Using this inequality, it can be shown that $g^{*}(h)(z)=\infty$ for the $g^{*}$ function of Definition 5. We shall show that this same example is also the example we want for bounded $D$ and the $g^{*}$ function of Definition 6 . To simplify matters, we shall use a translation and reflection of $v(x, y)$. Let $u(x, y)=v(x, 1-y)$ and let $\Gamma^{\prime}=\{(x, y):|x| \leq \alpha(1-y)\}$. Let $D$ be the unit ball in $R^{n+1}, x^{0}=0$, and $\mathbf{1}=(0, \ldots, 0,1)$. Then if $f=\left.u\right|_{\partial D}, f$ has a pole of order $(n+1) / 2$ at $\mathbf{1}$, and hence $f \in L^{p}(\partial D)$ for $p<2 n /(n+1)$. But we shall show that $g^{*}(f)(z)=\infty$. In what follows, we shall not distinguish a vertical direction and we will write $u(y)\left(y \in R^{n+1}\right)$ instead of $u(x, y)\left(x \in R^{n}, y \in R^{1}\right) . C_{n}$ will be a constant 
depending on $n$ whose value may change from line to line. Let $\gamma=\min \left(|z-\mathbf{1}|, \frac{1}{2}\right)$. Let $\Gamma(r)=\{y:|y|=r,|y-\mathbf{1}| \leq \beta(1-|y|)\}$, where $\beta>1$ depends solely on $\alpha$ and is chosen so that $\Gamma(r) \subset \Gamma^{\prime}$ for $\frac{1}{2} \leq r \leq 1$. Note that for $\frac{1}{2} \leq r \leq 1$, $\int_{\Gamma(r)} d \sigma \geq c_{n}(1-r)^{n}$, where $d \sigma$ is Lebesgue surface measure in $R^{n+1}$.

$$
\begin{aligned}
\left(g^{*}(f)(z)\right)^{2} & \geq C_{n} \int_{D}\left(|y|^{1-n}-1\right)(1-|y|)^{2}|y-z|^{-n-1}|\nabla u(y)|^{2} d y \\
& \geq C_{n} \int_{D}(1-|y|)\left(1+\cdots+|y|^{n-2}\right)|y|^{1-n}(1-|y|) \\
& \quad \times(1+|y|)|y-z|^{-n-1}|\nabla u(y)|^{2} d y \\
& \geq C_{n} \int_{D}(1-|y|)^{2}|y-z|^{-n-1}|\nabla u(y)|^{2} d y \\
& \geq C_{n} \int_{1-\gamma}^{1} \int_{\Gamma(r)}(1-r)^{2}(|y-\mathbf{1}|+|z-\mathbf{1}|)^{-n-1}|y-\mathbf{1}|^{-n-3} r^{n} d \sigma d r \\
& \geq C_{n} \int_{1-\gamma}^{1}(1-r)^{2}(\beta(1-r)+|z-\mathbf{1}|)^{-n-1}[\beta(1-r)]^{-n-3}(1-r)^{n} d r \\
& \geq C_{n}|z-\mathbf{1}|^{-n-1} \int_{0}^{1} u^{-1}(\beta+u)^{-n-1} d u=\infty,
\end{aligned}
$$

where we have switched to spherical coordinates in step 4 and made the substitution $u=(1-r) /|z-\mathbf{1}|$ in step 6 . This shows our inequality fails for $p<2 n /(n+1)$. By taking $n \rightarrow \infty$, we see that our a priori inequality holds only for $p \geq 2$.

NOTE ADDED IN PROOF. It has come to the author's attention that R. Bañuelos has proven several similar results also using probabilistic methods.

\section{REFERENCES}

1. D. L. Burkholder, Distribution function inequalities for martingales, Ann. Probab. 1 (1973), $19-42$.

2. J. L. Doob, Stochastic processes, Wiley, New York, 1953.

3. R. Durrett, Brownian motion and martingales in analysis, Wadsworth, Belmont, Calif., 1984.

4. R. F. Gundy and N. Th. Varopoulos, Les transformations de Riesz et les integrales stochastiques, C. R. Acad. Sci. Paris Sér. A 289 (1979), 13-16.

5. S. Kakutani, Two-dimensional Brownian motion and harmonic functions, Proc. Imp. Acad. Tokyo 20 (1944), 706-714.

6. K. E. Petersen, Brownian motion, Hardy spaces and bounded mean oscillation, Cambridge Univ. Press, New York, 1977.

7. E. M. Stein, Singular integrals and differentiability properties of functions, Princeton Univ. Press, Princeton, N.J., 1970.

8. __ Some results in harmonic analysis in $R^{n}$, for $n \rightarrow \infty$, Bull. Amer. Math. Soc. (N.S.) 9 (1983), 71-73.

9. __ Three variations on the theme of maximal functions, Proc. Conf. El Escorial, 1983 (to appear). 08544

Department of Mathematics, Princeton University, Princeton, NeW Jersey 